\title{
Editorial
}

\section{Nonlinear Partial Differential Equations in Mathematics and Physics}

\author{
Bo-Qing Dong, ${ }^{1}$ Caidi Zhao, ${ }^{2}$ Xiaohong Qin, ${ }^{3}$ Linghai Zhang, ${ }^{4}$ and Liangpan $\mathrm{Li}^{5}$ \\ ${ }^{1}$ School of Mathematical Sciences, Anhui University, Hefei 230601, China \\ ${ }^{2}$ College of Mathematics and Information Science, Wenzhou University, Wenzhou 325035, China \\ ${ }^{3}$ Department of Mathematics, Nanjing University of Science and Technology, Nanjing 210094, China \\ ${ }^{4}$ Department of Mathematics, Lehigh University, 14 East Packer Avenue, Bethlehem, PA 18015, USA \\ ${ }^{5}$ Department of Mathematics, Loughborough University, Loughborough LE11 3TU, UK
}

Correspondence should be addressed to Bo-Qing Dong; bqdong@ahu.edu.cn

Received 8 March 2015; Accepted 8 March 2015

Copyright (C) 2015 Bo-Qing Dong et al. This is an open access article distributed under the Creative Commons Attribution License, which permits unrestricted use, distribution, and reproduction in any medium, provided the original work is properly cited.

Nonlinear partial differential equations models in mathematics and physics play an important role in theoretical sciences. The understanding of these nonlinear partial differential equations is also crucial to many applied areas such as meteorology, oceanography, and aerospace industry. Nonlinear partial differential equations are the most fundamental models in studying nonlinear phenomena. This special issue is devoted to the analysis of partial differential models in fluid dynamics and related areas. It consists of eight papers.

Topics covered by this collection of papers include the analysis of nonlinear partial differential equations including Navier-Stokes-Poisson equations, DGH equation, reactiondiffusion equation, nonlinear pseudoparabolic equation, non-Newtonian fluid equations, nonlinear heat equation, and Navier-Stokes equations.

The paper by J. Liu and H. Liu is concerned with initial boundary value problem for 3-dimensional compressible bipolar Navier-Stokes-Poisson equations with densitydependent viscosities. When the initial data is large, discontinuous, and spherically symmetric, the global existence of the weak solution is obtained. The paper by Z. Guo and M. Zhao is concerned with the Dullin-Gottwald-Holm (DGH) equation with strong dissipative term. Some sufficient conditions to guarantee finite-time blowup of strong solutions are established. The paper by Z. Cao and Y. Lin deals with a variable-coefficient reaction-diffusion equation. For the three kinds of equations according to the variable coefficient in the process of solving the determining equations of Lie groups, the conservation laws corresponding to the symmetries obtained are considered. The paper by H. Di and Y. Shang investigates the nonlinear pseudoparabolic equation with a memory term. A finite-time blowup result by using the concavity method is proved. The paper by Z. Luo studies the optimal convergence rates for solutions of the monopolar non-Newtonian flows. By using the energy methods, the perturbed weak solution of perturbed system is proved to asymptotically converge into the solution of the original system with optimal rates. The paper by X. Hu is focused on the error estimates for solutions of the three-dimensional semilinear parabolic equation with initial data. Employing the energy methods and the Fourier analysis technique, the error between the solution of the semilinear parabolic equation and that of linear heat equation is obtained. The paper by Y. Yang deals with the algebraic time decay rate for weak solutions of the nonlinear heat equations with the strongly nonlinear term. An energy method is applied. The paper by J. Ren is concerned with the large time behavior of the weak solutions for three-dimensional globally modified Navier-Stokes equations. The optimal upper and lower decay estimates of the weak solutions for the globally modified Navier-Stokes equations are investigated.

\section{Acknowledgment}

We would like to express our sincere thanks to all the referees who generously devoted their time and effort reading and 
checking carefully all the papers and providing precious comments and recommendation.

Bo-Qing Dong

Caidi Zhao

Xiaohong Qin

Linghai Zhang Liangpan Li 


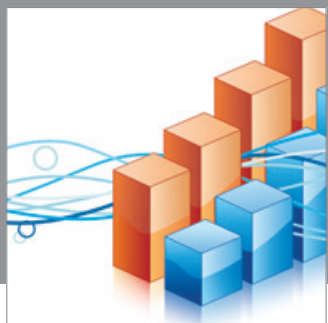

Advances in

Operations Research

mansans

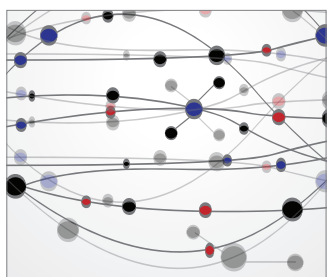

The Scientific World Journal
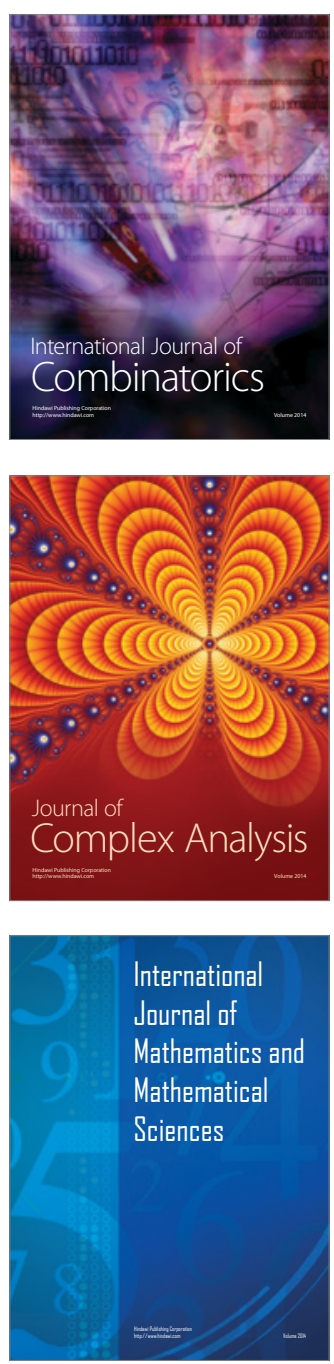
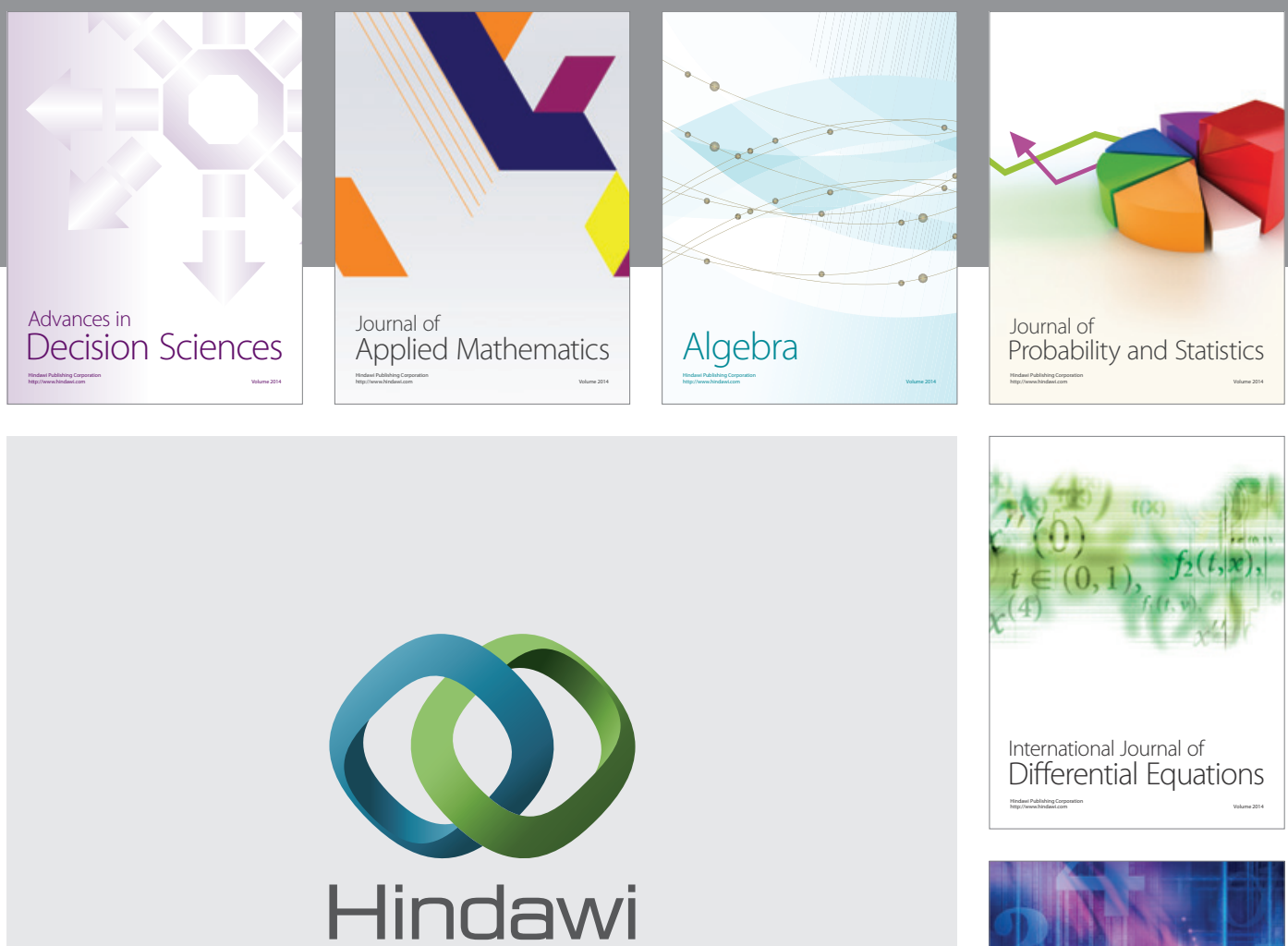

Submit your manuscripts at http://www.hindawi.com
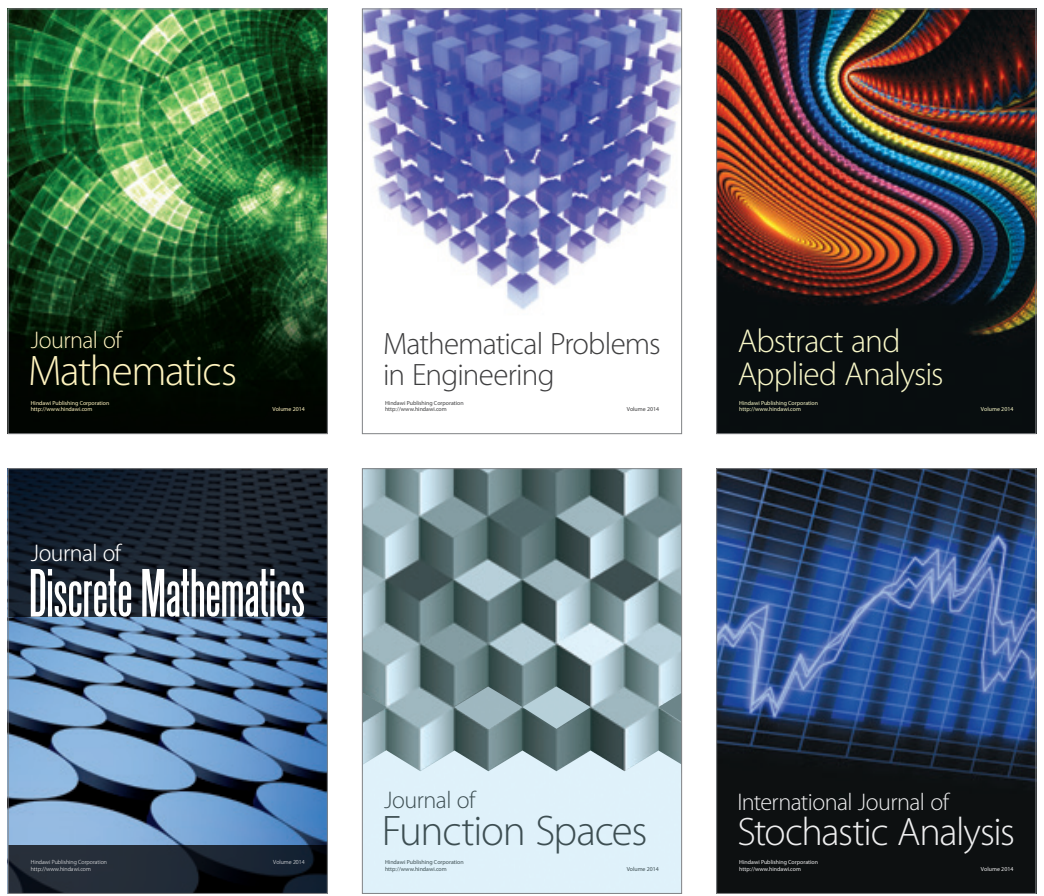

Journal of

Function Spaces

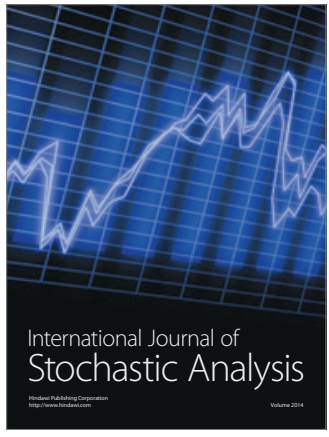

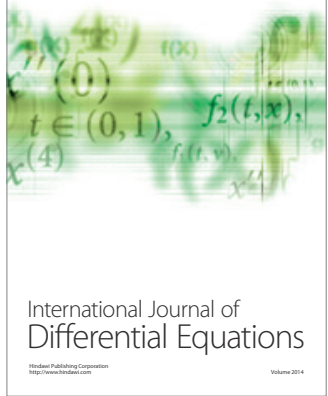
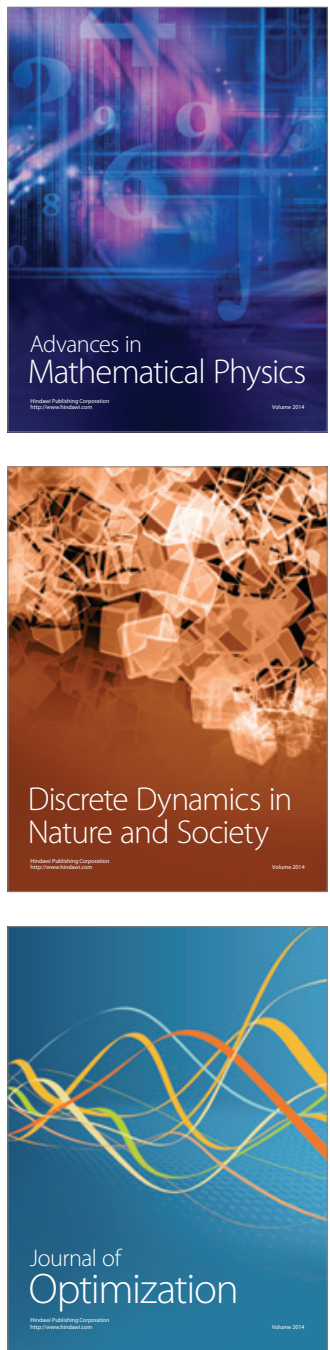